Ágora Rev. Cient. 2019; 06(02):e4

\title{
Efecto hepatoprotector del extracto hidroalcohólico de Desmodium molliculum ("manayupa") en ratas con intoxicación hepática inducida por paracetamol
}

\author{
Hepatoprotective effect of hydroalcoholic extract of Desmodium molliculum ("manayupa") in rats with \\ hepatic intoxication induced by paracetamol \\ Pamela Yanina Saucedo Estela ${ }^{1}$, Jhomaira Karen Tocto Céspedes ${ }^{1}$, Fidel Ernesto Acaro Chuquicaña ${ }^{1}$
}

\begin{abstract}
RESUMEN
Objetivo: Determinar el efecto hepatoprotector del extracto hidroalcohólico de Desmodium molliculum ("manayupa") a diferentes concentraciones (50, 500 y $800 \mathrm{mg} / \mathrm{kg}$ ), además de evaluar sus metabolitos activos. Materiales y Métodos: Se empleó extracto hidroalcohólico de D. molliculum en ratas Holtzman distribuidas de forma aleatoria en seis grupos $(n=5)$, las cuales recibieron los siguientes tratamientos, por diez días, vía peroral: grupos I y II: suero fisiológico $10 \mathrm{~mL} / \mathrm{kg}$; grupo III: silimarina $100 \mathrm{mg} / \mathrm{kg}$; grupo IV: 50 $\mathrm{mg} / \mathrm{kg}$; grupo V: $500 \mathrm{mg} / \mathrm{kg}$ y grupo VI: $800 \mathrm{mg} / \mathrm{kg}$ del extracto hidroalcohólico de D. molliculum. Al sexto día de tratamiento los grupos del II al VI recibieron paracetamol a $400 \mathrm{mg} / \mathrm{kg}$ vía peroral, con una hora de diferencia del tratamiento anterior, hasta completar los diez días. Resultados: De acuerdo a las dosis administradas se observó que el extracto a una concentración de $500 \mathrm{mg} / \mathrm{kg}$ y $800 \mathrm{mg} / \mathrm{kg}$ presentó mayor efecto hepatoprotector. Conclusiones: El extracto hidroalcohólico de la planta Desmodium molliculum ("manayupa") presenta un efecto hepatoprotector.
\end{abstract}

Palabras clave: hepatotoxicidad, paracetamol, Desmodium molliculum

\begin{abstract}
Objective: To determine the hepatoprotective effect of the hydroalcoholic extract of Desmodium molliculum ("manayupa") at different concentrations $(50,500$ and $800 \mathrm{mg} / \mathrm{kg})$, and also to evaluate its active metabolites. Materials and Methods: Hydroalcoholic extract of D. molliculum was applied to Holtzman rats randomized into six groups $(n=5)$, which received the following treatments, for ten days, via peroral: groups I and II: physiological serum $10 \mathrm{~mL} / \mathrm{kg}$; group III: silymarin $100 \mathrm{mg} / \mathrm{kg}$; Group IV: $50 \mathrm{mg} / \mathrm{kg}$; group V: $500 \mathrm{mg} / \mathrm{kg}$ and group VI: $800 \mathrm{mg} / \mathrm{kg}$ of the hydroalcoholic extract. On the sixth day of treatment, rats from groups II to VI received paracetamol at $400 \mathrm{mg} / \mathrm{kg}$ perorally, with one hour difference from previous treatment, until complete ten days. Results: According to the doses administered, it was observed that the extract of 500 $\mathrm{mg} / \mathrm{kg}$ and $800 \mathrm{mg} / \mathrm{kg}$ of the hydroalcoholic extract showed the largest hepatoprotective effect. Conclusions: The hydroalcoholic extract of Desmodium molliculum ("manayupa") showed an hepatoprotective effect.
\end{abstract}

Keywords: hepatotoxicity, paracetamol, Desmodium molliculum

${ }^{1}$ Universidad María Auxiliadora. Escuela Profesional de Farmacia y Bioquímica. Lima - Perú.

\section{INTRODUCCIÓN}

Las enfermedades hepáticas crónicas originan altos gastos al sistema sanitario, los cuales se van incrementando a medida que la hepatopatía va progresando, esto incorporado al hecho de que las afecciones del hígado crean un problema progresivo en todo el mundo (1). En el Perú, de acuerdo a un análisis realizado en todos los establecimientos de salud, la cirrosis hepática y diferentes afecciones graves del hígado, son las responsables de una cantidad de muerte de 21,3 por 100000 pobladores, ubicándose en el $9^{\circ}$ puesto, en la jerarquía de extensión entre el descenso más recurrente, siendo las personas de 20 a 65 años de edad las más afectados por estas afecciones (2).

La permanencia de las afecciones hepática en nuestro país, ha conducido a los habitantes a investigar y ver modos de procedimientos $\mathrm{y}$ medicación, produciendo suposiciones y tradiciones, por esta razón la cultura popular ha 
establecido medicaciones variables (medicina típica) y/o la automedicación que son repartidas por los pobladores sin distinción de escala educativa ni socioeconómica, lo que ha limitado a los pobladores a un alto riesgo de padecimientos (3). Así, existe un interés creciente para la búsqueda de tratamientos alternativos a través del empleo de plantas medicinales con propiedades hepatoprotectoras ante el incremento de las enfermedades referentes al hígado.

Por todo lo anteriormente expuesto, el objetivo del presente trabajo fue determinar el efecto hepatoprotector del extracto hidroalcohólico de Desmodium molliculum ("manayupa") en ratas Holtzman con intoxicación hepática inducida por paracetamol.

\section{MATERIALES Y MÉTODOS}

El estudio realizado es de carácter cuantitativo, prospectivo, longitudinal y además de ser experimental. Se empleó la técnica de inducción a hepatotoxicidad por paracetamol propuesta por Gibson y col.

\section{Obtención del extracto vegetal}

La muestra vegetal fue obtenida del departamento de Cajamarca, Provincia Cutervo, Distrito Pimpingos, Caserio el Progreso, durante el mes de marzo del año 2018. La ficha taxonómica fue certificada por el Museo de Historia Natural de la Universidad Nacional Mayor de San Marcos, según el sistema de clasificación de Arthur Cronquist (1988).

\section{REINO: PLANTAE \\ División: MAGNOLIOPHYTA \\ Clase: MAGNOLIOPSIDA \\ Sub Clase: ROSIDAE \\ Orden: FABALES \\ Familia: FABACEAE \\ Género: Desmodium \\ Especie: Desmodium molliculum}

La planta fue pesada y secada a $40{ }^{\circ} \mathrm{C}$ por 6 días, luego triturada a un tamaño de $3 \mathrm{~cm}$ para ser macerado en un frasco ámbar con solución hidroalcohólica al $70 \%$. La muestra fue filtrada y nuevamente secada en la estufa a $60{ }^{\circ} \mathrm{C}$.

\section{Análisis fitoquímico}

La identificación fotoquímica fue realizada por el método de Miranda, modificado por los autores. Este análisis fue del tipo cualitativo con la observación de la coloración para la identificación de los metabolitos secundarios (4).

\begin{tabular}{|c|c|c|}
\hline Reactivo & Reacción & Resultado \\
\hline $\mathrm{FeCl} 3$ & $\begin{array}{l}1 \mathrm{~mL} \text { extracto etanólico }+\mathrm{V} \text { gts } \\
\mathrm{FeCl} 3\end{array}$ & $\begin{array}{l}\text { Coloración } \\
\text { verde } \\
\text { azulado }\end{array}$ \\
\hline Shinoda & $\begin{array}{l}1 \mathrm{ml} \text { extracto etanólico }+(7 \\
\text { virutas de } \mathrm{Mg} \text { metalico }+\mathrm{V} \\
\text { gts } \mathrm{Hcl})\end{array}$ & $\begin{array}{l}\text { Coloración } \\
\text { roja }\end{array}$ \\
\hline $\mathrm{AlCl} 3$ & $\begin{array}{l}1 \mathrm{ml} \text { extracto etanólico }+V \\
\text { gotas } \mathrm{AlCl} 3\end{array}$ & $\begin{array}{l}\text { Fluorescenci } \\
\text { a amarilla } \\
\text { (luz U.V) }\end{array}$ \\
\hline $\begin{array}{l}\text { Dragend } \\
\text { orff }\end{array}$ & $\begin{array}{l}1 \mathrm{~mL} \text { extracto etanólico }+\mathrm{V} \text { gts } \\
\text { Rvo. Dragendorff }\end{array}$ & $\begin{array}{l}\text { Precipitado } \\
\text { rojo naranja }\end{array}$ \\
\hline Mayer & $\begin{array}{l}1 \mathrm{~mL} \text { extracto etanólico }+\mathrm{V} \text { gts } \\
\text { Rvo. Mayer }\end{array}$ & $\begin{array}{l}\text { Precipitado } \\
\text { blanco }\end{array}$ \\
\hline popoff & $\begin{array}{l}1 \mathrm{~mL} \text { extracto etanólico }+\mathrm{V} \text { gts } \\
\text { Rvo. Popoff }\end{array}$ & $\begin{array}{l}\text { Precipitado } \\
\text { amarillo }\end{array}$ \\
\hline Wagner & $\begin{array}{l}1 \mathrm{~mL} \text { extracto etanólico }+\mathrm{V} \text { gts } \\
\text { Rvo. Wagner }\end{array}$ & $\begin{array}{l}\text { Precipitado } \\
\text { pardo } \\
\text { oscuro }\end{array}$ \\
\hline $\begin{array}{l}\text { Sonnesc } \\
\text { hein }\end{array}$ & $\begin{array}{l}1 \mathrm{~mL} \text { extracto etanólico }+\mathrm{V} \text { gts } \\
\text { Rvo. Sonneschein }\end{array}$ & $\begin{array}{l}\text { Precipitado } \\
\text { amarillo } \\
\text { verdoso }\end{array}$ \\
\hline gelatina & $\begin{array}{l}1 \mathrm{~mL} \text { extracto etanólico + II gts } \\
\text { Rvo. Gelatina }\end{array}$ & $\begin{array}{l}\text { Precipitado } \\
\text { blanco }\end{array}$ \\
\hline
\end{tabular}

\section{Procesamiento de datos}

El procedimiento de análisis de datos se realizó mediante software estadístico SPSS 21.0 para Windows y Minitab versión en español, los cuales nos permitió elaborar cuadros de distribución de frecuencia, gráficos y calcular los datos estadísticos necesarios para el estudio.

\section{RESULTADOS}

Con respecto a la composición fitoquímica, se evidencio por el método cualitativo la identificación de flavonoides, alcaloides, taninos y fenoles, los cuales podrían estar involucrados con la actividad hepatoprotectora del extracto hidroalcohólico de "manayupa".

\begin{tabular}{|l|l|l|}
\hline $\begin{array}{l}\text { Metabolito } \\
\text { secundario }\end{array}$ & Reactivo & Resultado \\
\hline Flavonoides & Shinoda & POSITIVO \\
\hline Alcaloides & $\mathrm{AlCl}_{3}$ & POSITIVO \\
\hline & Mayer & POSITIVO \\
\hline Fenoles & Fopoff & POSITIVO \\
\hline Taninos & Gelatina & POSITIVO \\
\hline
\end{tabular}

Tabla 3. Estudio fitoquímico del extracto hidroalcoholico de D. molliculum ("manayupa") 
INVESTIGACIÓN ORIGINAL ORIGINAL RESEARCH
Efecto hepatoprotector del extracto hidroalcohólico de Desmodium molliculum ("manayupa") en ratas con intoxicación hepática inducida por paracetamol Saucedo Estela, Tocto Céspedes y Acaro Chuquicaña
Según los resultados se presenta la interpretación de acuerdo a cada una de las hipótesis formuladas (Tabla 1). Así, de acuerdo al valor de la significancia puede asumirse que los valores promedio son similares o no difieren de forma importante entre el grupo de control negativo, el grupo tratado con silimarina y con paracetamol, el grupo tratado con extracto hidroalcohólico de Desmodium molliculum en dosis de $50 \mathrm{mg} / \mathrm{Kg}$ con paracetamol, el grupo tratado con extracto hidroalcohólico de Desmodium molliculum en dosis de $500 \mathrm{mg} / \mathrm{Kg}$ con paracetamol y el grupo tratado con extracto hidroalcohólico de Desmodium molliculum en dosis de $800 \mathrm{mg} / \mathrm{Kg}$ con paracetamol. Los valores promedio son similares o no difieren de forma importante entre el grupo de control positivo y el grupo tratado con extracto hidroalcohólico de Desmodium molliculum en dosis de $50 \mathrm{mg} / \mathrm{Kg}$ con paracetamol. Puede asumirse la actividad similar entre los grupos comparados (Tabla 2).
Por otro lado, de acuerdo al valor de la significancia y a los conjuntos con grupos homogéneos se asume la actividad o efecto similar en el subconjunto 1 entre el grupo de control negativo, el grupo tratado con silimarina con paracetamol, el grupo tratado con extracto hidroalcohólico de Desmodium molliculum en dosis de $50 \mathrm{mg} / \mathrm{Kg}$ con paracetamol, el grupo tratado con extracto hidroalcohólico de Desmodium molliculum en dosis de $500 \mathrm{mg} / \mathrm{Kg}$ con paracetamol y el grupo tratado con Extracto hidroalcohólico de Desmodium molliculum en dosis de $800 \mathrm{mg} / \mathrm{Kg}$ con paracetamol. Se asume la actividad o efecto similar en el subconjunto 2 entre el grupo de control positivo y el grupo tratado con extracto hidroalcohólico de Desmodium molliculum en dosis de $50 \mathrm{mg} / \mathrm{Kg}$ con paracetamol.

\begin{tabular}{llc} 
HSD de Tukey & Pruebas post hoc de Comparaciones múltiples & \\
\hline \hline Tratamiento & Grupos de comparación & P.valor \\
\hline & Silimarina con Paracetamol & $\mathbf{0 , 9 4 7}$ \\
& Extracto en dosis $50 \mathrm{mg} / \mathrm{Kg}$ con paracetamol & $\mathbf{0 , 0 8 3}$ \\
Control negativo & Extracto en dosis $500 \mathrm{mg} / \mathrm{Kg}$ con paracetamol & $\mathbf{0 , 9 4 4}$ \\
& Extracto en dosis $800 \mathrm{mg} / \mathrm{Kg}$ con paracetamol & $\mathbf{0 , 9 6 0}$ \\
Control positivo & & $\mathbf{0 , 3 1 9}$ \\
\hline \hline
\end{tabular}

Tabla 1. Comparación entre los tratamientos y los controles negativo y positivo.

\begin{tabular}{lccc} 
HSD de Tukey & $\mathrm{N}$ & \multicolumn{2}{c}{ Subconjunto para alfa $=0.05$} \\
\hline \hline Tratamiento & & 1 & 2 \\
\hline Control negativo & $\mathbf{5}$ & $\mathbf{1 0 , 5 0 0 0}$ \\
Extracto en dosis $800 \mathrm{mg} / \mathrm{Kg}$ con paracetamol & $\mathbf{5}$ & $\mathbf{1 1 , 2 3 2 0}$ & \\
silimarina con paracetamol & $\mathbf{5}$ & $\mathbf{1 1 , 2 8 2 0}$ & \\
Extracto en dosis $500 \mathrm{mg} / \mathrm{Kg}$ con paracetamol & $\mathbf{5}$ & $\mathbf{1 1 , 2 9 4 0}$ & $\mathbf{1 3 , 0 2 6 0}$ \\
Extracto en dosis $50 \mathrm{mg} / \mathrm{Kg}$ con paracetamol & $\mathbf{5}$ & $\mathbf{1 3 , 0 2 6 0}$ & $\mathbf{1 4 , 8 9 0 0}$ \\
Control positivo & $\mathbf{5}$ & & $\mathbf{0 , 3 1 9}$ \\
Sig. & & $\mathbf{0 , 0 8 3}$ &
\end{tabular}

Tabla 2. Determinación de la efectividad de los tratamientos empleados 


\section{DISCUSIÓN}

La utilización de plantas medicinales para la prevención y/o tratamiento de enfermedades se ha incrementado en los últimos años, ya que su uso es muy recurrente entre los pobladores de bajos recursos, debido a que es la primera alternativa para curar cualquier tipo de enfermedad o dolencia, ya que no tienen acceso a los medicamentos. Por tal motivo, el presente estudio tiene como finalidad comprobar, con el rigor científico el efecto hepatoprotector del extracto hidroalcohólico de Desmodium molliculum ("manayupa") con la finalidad de garantizar y estandarizar su uso adecuado.

Se evidenció por el método cualitativo la identificación de flavonoides, alcaloides, taninos y fenoles, los cuales posiblemente sean los responsables de la actividad hepatoprotectora. Nuestros resultados son similares a los obtenidos por Chiu YJ, et al (2018), donde en los estudios fitoquímicos demostraron que los frutos del extracto etanólico de Polygonum orientale son ricos en flavonoides y son considerados como los principales componentes activos. Los flavonoides son una clase grande de compuestos fenólicos y constituyen uno de los grupos más grandes de metabolitos secundarios en las plantas. Además, estos compuestos protegen significativamente a las células frente a los efectos dañinos de las especies reactivas del oxígeno (ERO) tales como el oxígeno singlete, el superóxido, los radicales de peroxilo, los radicales hidroxilo y el peroxinitrito. El estrés oxidativo contribuye al desarrollo de enfermedades entre ellas el cáncer y la cirrosis hepática (5).

En el grupo control negativo (suero fisiológico), el hígado de los roedores se encontró en condiciones estables, pero fue inducido al daño hepático con el control positivo (paracetamol), la misma que se observa en la diferencias de medias $(-4,39000)$, mientras que la combinación de silimarima más paracetamol y el extracto hidroalcohólico de Desmodium molliculum a 500 y $800 \mathrm{mg} / \mathrm{Kg}$, revertió el daño hepático inducido. Fue menor la actividad hepatoprotectora a concentración de $50 \mathrm{mg} / \mathrm{Kg}$, quizás esta dosificación no es la recomendable debido al grado de toxicidad provocados a dosis superiores.

A una concentración de $500 \mathrm{mg} / \mathrm{Kg}$ se manifiesta en la observación macroscópica una reversión del daño en el hígado lesionado, Nuestras muestras son similares, aunque a dosis cercanas si son comparados con el estudio de Kokhdan P, et al (2017) del extracto etanólico de Stachys pilifera en tetracloruro de carbono inducido en ratas experimento a $400 \mathrm{mg} / \mathrm{Kg}$ y observó una mejoría en las lesiones hepáticas provocadas. Esta investigación determina que a esas dosis puede estar relacionada con sus propiedades antioxidantes (6).

A una concentración de $800 \mathrm{mg} / \mathrm{Kg}$ se observó una regeneración superior del hígado, demostrando así que nuestro estudio es similar a los resultados obtenidos, a concentraciones similares si comparamos con el estudio de Sifuentes R, et al (2018) del efecto hepatoprotector de la Cucurbita maxima (zapallo macre) en Rattus norvegicus tratadas con isoniacida, experimento a $800 \mathrm{mg} / \mathrm{Kg}$, donde se observó la reducción altamente significativa de la lesión del hígado, demostrando así que este estudio puede estar relacionado con sus propiedades hepatoprotectoras (7).

La administración de la dosis más alta del extracto hidroalcohólico de Desmodium molliculum $(800 \mathrm{mg} / \mathrm{Kg})$ llevó a una disminución de la lesión del hígado, alcanzando los valores del grupo III (silimarina). Estos resultados nos muestran que el extracto hidroalcohólico de Desmodium molliculum a una concentración de $800 \mathrm{mg} / \mathrm{Kg}$ tiene un efecto hepatoprotector y que podría ser más eficiente frente al daño producido por el paracetamol que la silimarina.

\section{CONCLUSIONES}

Se concluye que el extracto hidroalcohólico de Desmodium molliculum ("manayupa") presenta efecto hepatoprotector en ratas Holtzman con intoxicación hepática, inducida por paracetamol, a concentraciones de 500 y $800 \mathrm{mg} / \mathrm{Kg}$, resultado similar al grupo silimarina más paracetamol.

\section{AGRADECIMIENTOS}

Los autores agradecen a la Universidad María Auxiliadora por facilitarles el desarrollo del presente trabajo. Así mismo a los profesores Mg. Victor Humberto Chero Pacheco y Dr. Ruben Cueva Mestanza.

\section{AUTOR DE CORRESPONDENCIA}

Mg. QF. Fidel Ernesto Acaro Chuquicaña Escuela Profesional de Farmacia y Bioquímica. Universidad María Auxiliadora.

Dirección: Av. Canto Bello 431. Lima 36. Perú. Teléfono: 999417996

E-mail: eacaro farmaceutico@yahoo.es 


\section{REFERENCIAS BIBLIOGRÁFICAS}

1. Bustíos C, Dávalos $\mathrm{M}$, Román $\mathrm{R}$. Características epidemiológicas y clínicas de la cirrosis hepática en la unidad de hígado del HNERM Es-Salud. (Perú) 2007: 27(3); 238245.

2. Perú, Ministerio de Salud. Análisis de la situación de salud del Perú. Ministerio de Salud Dirección general de epidemiologia; 2010.

3. Osorio L, Patiño T, Tagle M, Huayanay L. Percepciones, conocimientos y actitudes sobre enfermedades hepáticas en adultos sanos que acuden a instituciones de salud de estrato A, B y C. Rev gastroenterol 2010; 30(21):126- 132.

4. Corral A, De la Paz J, Concepción E, Hernández R, López D. Tamizaje, Tecnología, control de calidad y farmacología del extracto fluido de Bouganvillea spectabilis Willd. Rev Cubana Plant Med. 1997; 2 (2): 19-25.

5. Chiua YJ, Chou SC, Chiu, CS. Hepatoprotective effect of the ethanol extract of Polygonum orientale on carbon tetrachloride-induced acute liver injury in mice. Journal of Food and Drug Analysis. 2018; 26 (1): 369-379

6. Kokhdan E, Ahmadi K, Sadeghi H, Sadeghi H, Dadgary F, Danaei N, Aghamaali MR. Hepatoprotective effect of Stachys pilifera ethanol extract in carbon tetrachloride-induce hepatotoxicity in rats. Pharm Biol. 2017 Dec;55(1):1389-1393.

7. Sifuentes Ramos, Franz Lomedik. Efecto hepatoprotector de cucurbita maxima en rattus norvegicus tratadas con isoniacida. Repositorio Institucional UNITRU.2018. 